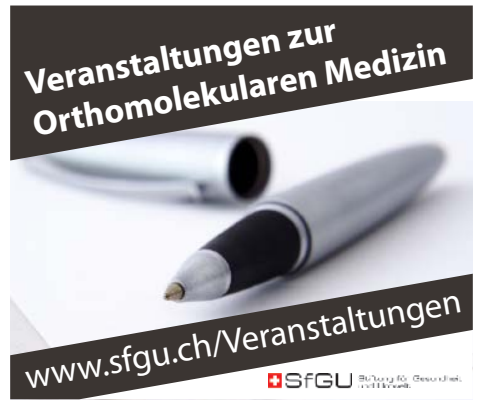

\title{
KARGER
}

Hier könnte Ihr Fort- und Weiterbildungsangebot stehen! Nutzen Sie SCHWEIZERISCHE ZEITSCHRIFT FÜR GANZHEITSMEDIZIN zur effektiven Kommunikation mit Ihrer Zielgruppe! So schaffen Sie die besten Voraussetzungen sowohl gedruckt als auch online gesucht und gefunden zu werden.

Informationen und Angebote bei:

Stefan Diekmann

GYNÄKOLOGIE (CUpdate Refresher

$$
\text { 8. - 9. Mai } 2013
$$

HÄMATO/ONKO (C) Update Refresher

$$
\text { 9. Mai } 2013
$$

DIAGNOSTIK ( U UPDATE ReFRESHER

$$
\text { 10. - 11. Mai } 2013
$$

ANÄSTHESIE UNDINTENSIVMEDIZIN (C) UPDATE ReFRESHER 18. - 19. Juni 2013

PSYCHIATRIE (C) Update Refresher 18. - 19. Juni 2013

APOTHEKER ( U UDAte Refresher 20. - 21. Juni 2013

\section{INNERE MEDIZIN}

(C)UPDATE KOMPAKT

10. - 11. Mai 2013

\section{ALLGEMEINE INNERE MEDIZIN}

(CUpdate Refresher

12. - 15. Juni 2013

\section{Veranstaltungsort}

Technopark Zürich 